\title{
Energy Conservation Protocol using Sleep Scheduling in WSN
}

\author{
Savita D. Gavande \\ Student \\ Flat No. A-9/12,Kunal Residency,Thergaon,Pune- \\ 411033
}

\begin{abstract}
Wireless Sensor Network is made-up of large number of sensor node and one or more sink node. Sensor nodes are small in size and low in cost. Sensor network are equipped with sensor devices and powered by small size batteries. Since each sensor node has low energy, these nodes are usually put to sleep mode to conserve energy, and this helps to increase the network lifetime. Sleep scheduling algorithm should take care that data continuously routed form sensor node to sink node. In this paper we are going discuss sleep scheduling for sensor network using energy conservation protocol and tree integrated with proposed sleep scheduling algorithm. The root node in tree is sink node and other sensor nodes are branch nodes and leaf nodes. The leaf nodes are always put to sleep mode and branch nodes are remain awake. This ensures that path must exist from any sensor node to sink node. The tree is periodically reconstructed considering the remaining energy of each node by checking balance energy consumption of nodes, and removes the failed nodes from the tree. This proposed approach greatly minimize average energy consumption rate of all sensor node as we are putting more number of nodes in sleep mode. In this paper, we are proposing a novel Efficient Energy-Aware Routing Protocol (EEAR) to increase lifetime of sensor network by minimizing energy consumption and balancing load between all the sensor nodes. We are also going to discuss simple but efficient approach named intra cluster coverage to handle area coverage problem.
\end{abstract}

\section{Keywords}

Energy Conservation, Wireless Sensor Network (WSN).

\section{INTRODUCTION}

Wireless Sensor network is a collection huge number of selforganized sensor nodes which periodically monitors data from surrounding and send it to sink node. WSN is widely used for target tracking in military, traffic monitoring, Intrusion and fire detection. Sensor nodes are small in size contains three units Sensor unit which sense data from surrounding, processing unit for data processing and data storage and transceiver unit which transmit data form sensor node to sink node and receive data form sink node. Sensor nodes are deployed in hostile areas so it is not possible to provide continuous energy supply to nodes so they are usually powered by battery with limited energy.

Due to constraints imposed by the available supply of energy each sensor node has limited computation power and memory. Major task of sensor node is to sense and record data for surrounding and send it to sink node if necessary. Sometimes sink node may also send data to sensor nodes though broadcast messages as well as sensor nodes may need to communicate with each other. In order to increase lifetime of the network, it is essential to reduce the consumption of energy by each nodes.

\author{
Santosh Kumar Biradar \\ Assistant Professor, \\ Dr.D.Y. Patil College of Engineering, Ambi, Pune
}

In addition, it is also necessary to ensure that the average rate of consumption of energy by each node is also the same to balance energy among each node. This would ensure that the connectivity needed to transmit data from a sensor node to sink node can always be maintained. A third requirement of WSNs for applications such as tracking of intruders, detection of fire etc. is that the delay to transmit data from sensor node to the sink should be as less as possible. These are complex set of requirements which a routing protocol for wireless sensor networks needs to fulfill. Moreover, the transceiver is the major unit that consumes lots of energy in each sensor node even when it is idle. Therefore, sensor nodes are usually put to sleep if they are not required to transmit data and/or sense environment, and the challenge is to integrate sleep scheduling scheme with routing protocols for WSNs so that the objective of routing protocols as given above are also met. We assume that the transceiver, processor, and sensing units can be put to sleep independently and when we say that the sensor node is put to sleep(transceiver and the processor are put to sleep). The sleep scheduling algorithm is allied independently to sensing units to ensure sensing coverage. In this paper, we propose a novel sleep scheduling scheme using a tree, and an energy aware routing protocol that is appropriately integrated with the above sleep scheduling scheme with a view to meet the objectives for routing protocols as given above.

\section{LITERATURE SURVEY}

There exists a considerable research effort for the development of routing protocols in WSNs. The development of these protocols is based on the particular application needs and the architecture of the network. However, there are several factors that should be taken into consideration when developing routing protocols for WSNs. Energy efficiency is the most important among these factors, since it directly affects the lifetime of the network. There have been a few efforts in the literature pursuing energy efficiency in WSNs.

Greedy routing approach has been utilized by many researchers for making decisions in order to establish links for routing paths. Greedy Perimeter Stateless Routing (GPSR)[1] employs position of the router and packet's destination to forward the data packet. This algorithm considers its neighbors for making routing decisions. When it is not possible to make a decision by using its neighbors, then it makes use of perimeter of the region to forward the packet to the selected node. Greedy routing decisions are taken by Energy Aware Greedy Routing (EAGR) algorithm depending upon residual node energy [2]. This paper presents an algorithm which makes use of residual energy at each neighboring node before selecting the next hop for its new route. EAGR approach not only provides higher throughput but also increases the lifetime of the network. Moreover, it balances the energy consumption of the nodes by choosing the next node with the highest residual energy. As part of its 
strategy, every node in the network knows its location and energy level besides keeping record of the location and energy level of its neighbors. The criterion used by the algorithm is that the transmitting node forwards the packet to that neighbor which has the highest residual energy level and whose distance is equal to or less than the average distance of all its neighbors. Geographic Adaptive Fidelity (GAF) is an energy aware algorithm primarily for ad hoc networks. To conserve energy of nodes, it switches off the redundant nodes without affecting reliability and performance of the network. A similar methodology has also been employed in Self Organizing Activity Node Density (SAND) approach. It enhances the routing and sensing fidelity by reducing the node activity. It switches off the radio when there is no communication in the network in order to conserve energy both in idle and overhearing state. SAND uses peer-to-peer paradigm and provides neighborhood communication for sensor resolution

In [3] Low Energy Adaptive Clustering Hierarchy (LEACH), a hierarchical protocol in which most nodes transmit to cluster heads, is presented. The operation of LEACH consists of two phases, The Setup Phase: In the setup phase, the clusters are organized and the cluster heads are selected. In every round, a stochastic algorithm is used by each node to determine whether it will become a cluster head. If a node becomes a cluster head once, it cannot become a cluster head again for $\mathrm{P}$ rounds, where $\mathrm{P}$ is the desired percentage of cluster heads. The Steady State Phase: In the steady state phase, the data is sent to the base station. The duration of the steady state phase is longer than the duration of the setup phase in order to minimize overhead.

In [4], a centralized routing protocol, called Base-Station Controlled Dynamic Clustering Protocol(BCDCP), which distributes the energy dissipation evenly among all the sensor nodes to improve the network lifetime, and its average energy savings are presented. The base station receives the residual energy of each node, and then, it computes the average energy level of all the nodes. Then, it elects as candidate cluster heads a number of nodes, which have a higher residual energy than this value. This protocol provides balanced energy consumption. However, the selection of the node with the highest energy as a cluster head at a round may cause the other nodes to spend more energy to send data to this node. The selection of a node that allows the other nodes in the cluster to spend less energy is a better solution.

Ye, Chen, Lu, and Zhang [5] have proposed an algorithm, called Minimum Cost Forwarding Algorithm (MCFA), that sets up a back off based cost field to find the optimal cost path from all the nodes to the sink. Once the field is established, the message, carrying dynamic cost information, flows along the minimum cost path in the cost field. This protocol consists of two phases. First phase is a setup phase for setting up the cost value in all nodes. In the second phase, the source broadcasts the data to its neighbors. reduce the number of broadcast

messages, the MCFA was modified to run a backoff algorithm at the setup phase. The backoff algorithm dictates that a node will not send the updated message until backoff. All the aforementioned protocols try to minimize the energy consumption using different algorithms. These algorithms offer a good solution, since they select the node with the higher residual energy in the cluster as the cluster head for the next round. However, this does not assure the maximum prolongation of the overall network lifetime. Therefore, if the node with the highest residual energy is a node located at the side of the cluster, this can lead other nodes to spend considerable amounts of energy to reach that node, which cannot be energy efficient for the entire network. This is the reason we propose a protocol that elects as cluster heads nodes that minimize the total energy consumption in a cluster.

\section{PROPOSED SYSTEM 3.1 Problem Definition}

WSN consist of small and low cost sensor node powered by small batteries each sensor. Node has limited energy. Energy aware routing protocol with which conserves the energy for WSNs. The core of the routing protocol is efficient construction of the broadcast tree with two paths from each node towards the sink to ensure fault-tolerance. The tree is reconstructed at the beginning of each period so that none of these nodes die before other nodes. Consecutive packets are routed through alternative path to reduce traffic in individual's paths. Leaf node sleep mechanism is highly energy efficient as more number of nodes are able to sleep and this helps to prolong the network lifetime. Construction of broadcast tree such that no of broadcast is as minimum as possible. After the construction of the tree is complete, each node can identify itself either as leaf node of the tree or as internal node of the tree. The leaf nodes are put to sleep and internal nodes remain awake. A node wishing to transfer data to the sink can send it along the edges of the tree towards the sink. The tree is constructed in such a way that the internal nodes have higher remaining energy compared to the other nodes. This is required to ensure that the consumption of energy by all nodes remains balanced. The tree is reconstructed periodically to ensure balanced consumption of energy by all the nodes.

\subsection{System Architecture}

The routing protocol proposed is intended for WSNs in which sensor nodes are static. Beside the applications running in the WSN require that the information gathered by the sensor nodes have to be transmitted immediately to the sink. Furthermore, it is also assumed that each node has a unique ID, and the communication between neighboring nodes is symmetric and bidirectional. At the same time it is also assumed that the clocks of the sensor nodes in the WSN are synchronized, so that nodes can be woken up nearly at the same time, and they can execute the protocol.

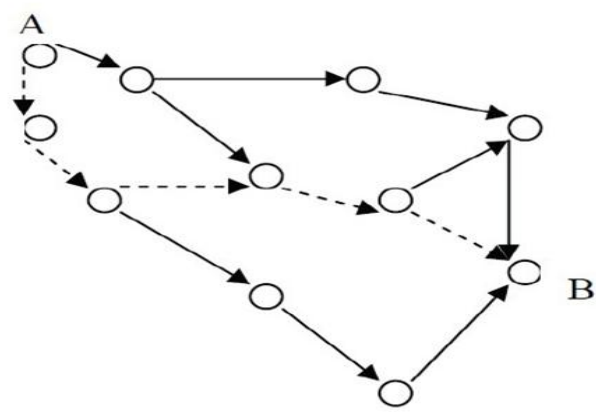

Fig 1: proposed System architecture

\subsection{Implementation Procedure}

As WSN consist of large number of sensor network the steps to acquire and process in WSN follows.

Step 1: The sink node is constructed.

Step 2: All the sensor nodes are constructed.

Step 3: By sending advertisement messages the construction of Broadcast tree is done. 
Step 4: Choose an appropriate Sensor node and select a message to send from sensor node to sink node.

Step 5: Click on send Button to send a message to sink node.

Step 6: Send a message through appropriate nodes to Sink node.

Step 7: Reduce the energy of all the nodes through which message is transferred.

Step 8: Plot a graph indicating energy reduction of all the nodes including sink node.

\subsection{Experimental Evolution}

In order to evaluate the performance of system performed. The network architecture considered is the following:

- A fixed base station(sink node) is located away from the sensor field.

- The sensor nodes are energy constrained with homogeneous initial energy allocation.

- Each sensor node senses the surroundings at a fixed rate and always has data to send to the base

- Station (data are sent if an event occurs).

- The sensor nodes are assumed to be stationary. However, the protocol can also support

- $\quad$ Node mobility.

- The network is homogeneous, and all the nodes are equivalent, i.e., they have the same configuration.

- Computing and communication capacity.

- The physical location of nodes is not known in advance.

- The transmitter can adjust its amplifier power based on the transmission distance.

The aforementioned network architecture is typical of numerous applications of hierarchical WSNs, such as in environment and habitat monitoring, surveillance and reconnaissance, home automation, biomedical applications, object tracking, traffic control, fire detection, inventory control, agriculture, machine failure diagnosis and energy management [6].

In a actual use of the proposed protocol, it may be used in a fire monitoring system in a set of buildings where the sensors in each building are grouped in the same cluster that send the data to the cluster of the next building.

\section{RESULT}

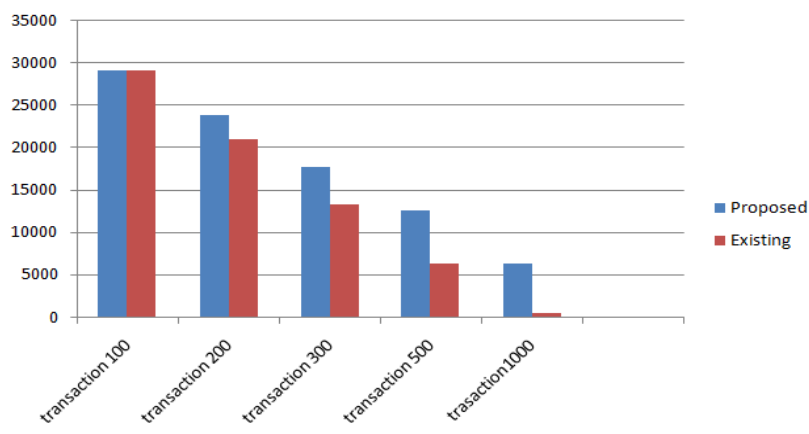

Fig 2: Energy level after transactions
In given figure 2 shows the energy level and $\mathrm{x}$ shows the number of operation of transmission as well as receiving between sink and radar nodes. So we can analyze our proposed system and algorithms are greatly increased the system performance and network live state-owned.

\section{CONCLUSION AND FUTURE WORK}

We have presented an energy aware routing procedure with sleep planning for WSNs. The core of the routing protocol is the efficient construction of the broadcast tree with two paths from each node towards the sink, and with higher outstanding energy at each internal node of the tree. The tree is restored at the start of each period so that none of these nodes die before other nodes, which means that all nodes will different around the same time. Repeated packets are routed through alternative path to reduce traffic in individual paths. Leaf node sleep mechanism is highly energy resourceful as more number of nodes stands able to sleep, and this helps to delay the network life-time. We have evaluated the performance of our procedure through simulation studies for changed number of nodes and rounds. Simulation results show that data packet Transfer in our multipath procedure is more than that using unique path, and energy consumption of nodes is also balanced. Comparison with GSP shows that our protocol has more number of snooze nodes, and therefore provides longer network lifetime. We have used very high data rate in our simulation readings. Future work includes adaptively adjusting the period of tree rebuilding depending on the input data rate with a view to further increase the network lifetime.

1. We can add interference detection system in current assignment

2. We can provide mobile alerts for sender when the file sent from sender is received at destination end.

3. We are fixed only on energy aspects but we can direct this Project towards resourceful routing of less similarly.

\section{REFERENCES}

[1] L. Wang and Y. Xiao, A survey of energyefcientschedul- ing mechanisms in sensor networks, Mob. Netw.Appl., vol. 11, no. 5, pp. 723740, 2006.

[2] Cc2420 data sheet, http://www.stanford.edu/class/cs244e/ papers/cc2420.pdf.

[3] Heinzelman, W.; Chandrakasan, A.; Balakrishnan, H. Energy-Efficient Communication Protocolfor Wireless Microsensor Networks. In Proceedings of the 33rd Hawaii International Conferenceon System Sciences, Hawaii, HI, USA, 2000; pp. 1-10.

[4] Muruganathan, S.; Ma, D.; Bhasin, R.; Fapojuwo, A. A Centralized Energy-Efficient Routing Protocol for Wireless Sensor Networks. IEEE Radio Commun.2005, $43,8-13$.

[5] F. Ye, A. Chen, S. Lu, and L. Zhang, "A scalable solution to minimum cost forwarding in large sensor networks," in Computer Communications and Networks, 2001. Proceedings. Tenth International

[6] Nikoletseas, S.; Spirakis, P.G. Probabilistic Distributed Algorithms for Energy Efficient Routing and Tracking in Wireless Sensor Networks. Algorithms 2009, 2, 121157. 